\title{
MRI can reveal metabolic changes in lily bulbs in vivo during dormancy release
}

\author{
HAN HaoJun ${ }^{1,2} \&$ YI MingFang ${ }^{1 *}$ \\ ${ }^{1}$ Department of Ornamental Horticulture and Landscape Architecture, China Agricultural University, Beijing 100193, China; \\ ${ }^{2}$ Fushun Teachers College, Fushun 113006, China
}

Received April 17, 2012; accepted September 27, 2012; published online October 31, 2012

\begin{abstract}
The factors influencing dormancy release in lily bulbs strongly affect commercialization success, but the mechanism of dormancy release is still unclear. Magnetic resonance imaging (MRI) can detect changes in morphology and water status in a living plant bulb and aid in investigating release factors. To evaluate whether MRI could be used to detect intra-bulb metabolic changes during the dormant period in Oriental Lilies (Lilium 'Sorbonne'), a series of MRI and sugar concentration measurements were performed weekly on bulbs stored for 11 weeks at $4^{\circ} \mathrm{C}$. The image quality of intra-bulb structure obtained using $T_{1}$-weighted imaging was superior to that obtained using $T_{2}$-weighted imaging and had a higher signal-to-noise ratio $(0.97 \pm 0.01)$. Magnetization transfer ratio values for the bud and basal plate declined during the first eight weeks of cold storage $(P>0.05)$, and were well correlated with concentration of soluble sugar in the bud $\left(R^{2}=0.95\right)$ and basal plate $\left(R^{2}=0.93\right)$. Thus, MRI can serve as a valuable tool for observation and analysis of dynamic morphological and metabolic changes in vivo during dormancy release. This information is potentially useful as a guide in the improvement of horticultural product quality.
\end{abstract}

transverse and longitudinal relaxation times, magnetization transfer ratio (MTR), soluble sugar, magnetic resonance imaging (MRI), Lilium oriental hybrids, dormancy release

Citation: Han H J, Yi M F. MRI can reveal metabolic changes in lily bulbs in vivo during dormancy release. Sci China Life Sci, 2012, 55: 1002-1006, doi: $10.1007 / \mathrm{s} 11427-012-4394-8$

Dormancy release in Oriental Lilies, which are perennial plants, generally requires a minimum of eight weeks at low temperatures $\left(4^{\circ} \mathrm{C}\right)$. Traditional in vitro methods can detect morphological and metabolic changes in lily bulbs at certain time points, which can then be verified by blocking development. These experiments have shown that the process is affected by many complicated factors. However, because in vitro methods do not provide continuous monitoring, the exact mechanism of lily bulb development is still unclear [1-7].

Magnetic resonance imaging (MRI) enables non-invasive, longitudinal assessment of the water status of localized tissues [8-10]. It has been used to study water status of flower

*Corresponding author (email: ymfang@cau.edu.cn) bulbs, providing insight into bulb development [11,12], and to investigate disorders such as bud abortion [13]. In plant studies, spin-lattice relaxation time $\left(T_{1}\right)$ and spin-spin relaxation time $\left(T_{2}\right)$ are often used as indicators of water status as they are primarily correlated with water content and mobility [14-16]. In addition, the magnetization transfer effect is applied to quantitatively measure the amount of magnetization transfer from bound to free water in living plant tissues [17].

We searched for the optimal MRI sequence for morphology monitoring and changes in water correlation coefficients. Dynamic changes in $T_{1}, T_{2}$ parameters and magnetization transfer ratio (MTR) were summarized during lily bulb dormancy break, and correlations between water molecule relaxation, soluble sugar, and starch were investigated. 
Our study provides a technological foundation for in vivo investigations of the mechanism for dormancy release in lily bulbs.

\section{Materials and methods}

\subsection{Plant material}

Oriental Lilies (Lilium 'Sorbonne') were harvested in mid-December 2011 and placed in cold storage $\left(4^{\circ} \mathrm{C}\right.$ and $60 \%$ relative humidity) for 11 weeks. Mean circumference, height, and weight of harvested bulbs $(n=30)$ was $(16.12 \pm$ $1.2) \mathrm{cm},(4.38 \pm 0.65) \mathrm{cm}$, and $(2.41 \pm 4.2) \mathrm{g}$, respectively. Each week, 12 lily bulbs were chosen and randomly divided into two groups: one for carbohydrate analysis $(n=6)$, and the other for MRI monitoring $(n=6)$. We focused our studies on the following bulb regions: basal plate, bud, exterior scale, middle scale and interior scale.

\subsection{Magnetic resonance (MR) imaging}

MR scanning of individual bulbs was performed using a Siemens Magnetom Trio Tim system (Siemens Medical Solutions, Erlangen, Germany) interfaced to a head coil at 3.0 Tesla. Coronal plane images and relaxation measurements were obtained from entire bulbs on a Siemens NUMARIS/4 workstation. $T_{1}$ maps were generated from a series of $T_{1}$-weighted MR images, with repetition time $(T R)=500 / 1000 \mathrm{~ms}$ and echo time $(T E)=8.9 \mathrm{~ms}$. $T_{2}$-weighted images were acquired using the following parameters: $T R=2000 \mathrm{~ms}, T E=33.3 / 66.6 / 99.9 \mathrm{~ms}$, flip angle $=180^{\circ}$, band width $=558 \mathrm{~Hz}$, field of view $=210 \mathrm{~mm} \times 210 \mathrm{~mm}$, slice thickness $=2 \mathrm{~mm}$, and matrix $=320 \times 240$. The procedure used for each bulb consisted of acquiring irradiation-on and irradiation-off images for two perpendicular planes across the center of the bulb. For the scanning sequence used to obtain MTR values, $T R$ was set to $2300 \mathrm{~ms}$ and $T E$ to $9.8 / 79 \mathrm{~ms}$. MTR values were derived pixel-by-pixel from proton density-weighted images, with and without preceding offresonance irradiation. The quantitative equation used was $\mathrm{MTR}=(M-M s) / M$, where $M s$ and $M$ are the image intensities in the presence and absence of the off-resonance irradiation, respectively.

\subsection{Carbohydrate analysis}

Starch and soluble sugar concentrations were determined from randomly-selected bulbs using the anthrone colorimetric technique. Basal plate, bud, exterior scale, middle scale and interior scale regions were assayed.

\subsection{Data analysis}

Mean values of $T_{1}, T_{2}$, and MTR parameters were calculated from MR images by defining regions-of-interest (ROI), which were localized in the targeted subregions of the bulb and connected pixels within a narrowly defined intensity range, typically $10 \%$ around the mean. Mean parameter values calculated for each bulb were then averaged over the population in each group. $T_{1}$ and $T_{2}$ were calculated according to the following equation:

$$
S=M_{0}\left(1-2 \mathrm{e}^{-\frac{T R-\frac{T E}{2}}{T_{1}}}+\mathrm{e}^{-\frac{T R}{T_{1}}}\right) \cdot \mathrm{e}^{-\frac{T E}{T_{2}}},
$$

where $S$ is the signal intensity of the ROI measured on MR images and $M_{0}$ is the net magnetization vector. The signal-to-noise ratio (SNR) of the MR image was used to assess the image quality:

$$
\mathrm{SNR}=\left(S_{\text {means(bud) }}-S_{\mathrm{b} \text { (background) }}\right) / S D_{\text {(bud) }} \text {, }
$$

where $S_{\text {means(bud) }}$ and $S_{\text {b(background) }}$ represent the average pixel intensity of the bud and background, respectively. $S D_{\text {(bud) }}$ is the average signal variance for all pixels in the bud.

Concentrations of soluble sugar $\left(C_{\mathrm{ss}}\right)\left(\mathrm{mg} \mathrm{g}^{-1} \mathrm{FW}\right)$ and starch $\left(C_{\mathrm{s}}\right)\left(\mathrm{mg} \mathrm{g}^{-1} \mathrm{FW}\right)$ were estimated according to eqs. (3) and (4):

$$
\begin{gathered}
C_{\mathrm{ss}}=\frac{C \cdot\left(\frac{V}{a}\right) \cdot n}{W \cdot 1000} \times 100, \\
C_{\mathrm{s}}=\frac{C \cdot\left(\frac{V}{a}\right) \cdot n \times 0.9}{W \cdot 1000} \times 100 .
\end{gathered}
$$

Here $C$ was the amount of glucose $(\mu \mathrm{g})$ in the sample based on a standard curve, $V$ was the sample extraction volume $(\mathrm{mL}), a$ was the volume of sample solution $(\mathrm{mL}), n$ was the dilution ratio, and $W$ was the sample weight $(\mathrm{mg})$. The glucose conversion coefficient used for starch was 0.9 .

Results were presented as means \pm standard deviation $(S D)$. Linear correlation analysis between parameters was performed using SPSS 13.0 software, and the square of linear correlation coefficient $\left(R^{2}\right)$ was used to evaluate goodness of linear fit.

\section{Results}

\subsection{Carbohydrate changes during cold storage}

During storage, bulbs were analyzed biweekly for carbohydrates. Bulbs were dissected into basal plates, buds and scales. Starch concentration in both scales and buds decreased markedly in the first four weeks of cold storage, although the reduction was less apparent in basal plate compared with other regions. The decline in starch content was less rapid from the fourth through the eighth week, after which values remained relatively stable (Figure 1A). On the eighth week, the percentages of starch remaining compared with original levels were as follows: basal plate 

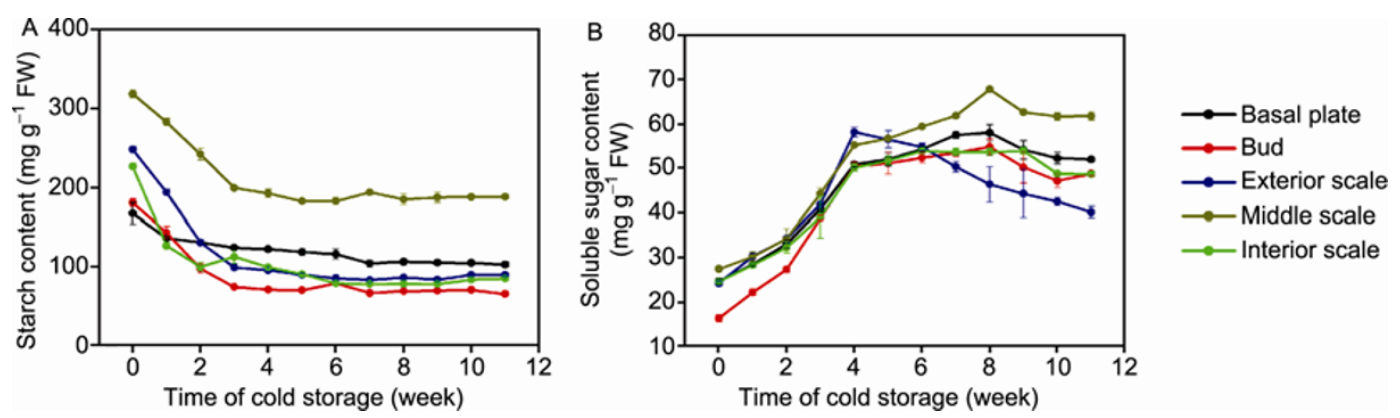

Figure 1 The content-time profiles of starch (A) and soluble sugar (B) were analyzed during 11 weeks of storage at $4{ }^{\circ} \mathrm{C}$. In the 8 th week, the percentages of starch remaining compared with original levels were as follows: basal plate $(36.19 \%)$, bud $(61.72 \%)$, exterior scale $(65.12 \%)$, middle scale $(41.92 \%)$ and interior scale $(65.43 \%)$. The decrease in starch content was accompanied by an increase in soluble sugar concentration.

(36.19\%), bud $(61.72 \%)$, exterior scale $(65.12 \%)$, middle scale $(41.92 \%)$ and interior scale $(65.43 \%)$.

The starch decrease was accompanied by an increase in soluble sugar concentrations $C_{\text {[sugar] }}$ (Figure 1B). In our study, $C_{\text {[sugar] }}$ values experienced their highest growth rates during the first four weeks of cold storage, generally reaching peak values by the eighth week and then gradually declining (Figure 1B). This trend was obvious in the basal plate, bud and middle scale. During the eighth week, the rates of increase were as follows: basal plate (2.3-fold), bud (3.3-fold), exterior scale (1.9-fold), middle scale (2.3-fold), and interior scale (2.2-fold).

\subsection{MRI measurements}

$T_{1}$ and $T_{2}$ values showed very little change over the storage period and the small amount of variation was not significant (Figure $2 \mathrm{~A}$ and $\mathrm{B}$, respectively). Although $T_{1}$ and $T_{2}$ values in all parts of the lily bulb should have theoretically been shorter, our results revealed no significant changes. $T_{1}$ and $T_{2}$ were not well correlated with changes in $C_{\text {[sugar] }}$.

Mean MTR values for various ROI are shown in Figure 2C. Decreases in MTR values were not uniform across sampled regions, even though the overall trend was a decrease during early cold storage. Coefficients of variation for MTR values in the bud (39.67\%) and basal plate $(66.66 \%)$ were significantly higher than for those in the outer layer scales (13.23\%). Declines in MTR values were more apparent in the basal plate and bud than in other regions. During cold storage, a significant change in MTR values was observed over time for the bud $(F=218.06$, $P<0.05)$ and basal scale $(F=138.14, P<0.05)$.

When we analyzed MTR values, we found significant variations between different regions; for example, the lowest values for exterior scale were observed after five to six weeks of cold storage compared with eight weeks for the bud, basal plate, and middle scale and nine weeks for the interior scales (Figure 2C). Relationships between MTR values and $C_{\text {[sugar] }}$ in the bud and basal plate were examined to test our hypothesis that active metabolic activities in these areas are more likely to reflect activation of the dormancy release period. MTR values in the basal plate varied sig- nificantly during cold storage and decreased to a minimum point during the eighth week, and then increased continuously afterward. This was consistent with the observed change in $C_{\text {[sugar] }}$. There was a significant correlation between $C_{\text {[sugar] }}$ and MTR for both bud $\left(R^{2}=0.95\right.$, Figure 2D) and basal plate $\left(R^{2}=0.93\right.$, Figure $\left.2 \mathrm{E}\right)$.

\subsection{Morphological analysis}

From the two sequences image series obtained for bulb internal morphology, the best image quality of the internal structures was obtained using $T_{1}$-weighted images ( $\left.T_{1} \mathrm{WIs}\right)$. MRI signals were intense in the basal plate and bud than in peripheral bulb regions (Figure $3 \mathrm{~A}$ and $\mathrm{B}$ ). The poor image quality on $T_{2}$-weighted images $\left(T_{2} \mathrm{WIs}\right)$ gave rise to a lower SNR than that of the $T_{1}$ WIs $(t=21.88, P<0.01)$. The internal morphology of lily bulb in vertical section is shown in Figure $3 \mathrm{C}$.

\section{Discussion}

Starch is the most abundant nonstructural carbohydrate in lily bulbs. The starch content of lily bulb scales was highest in the beginning of cold storage, followed by a decrease during storage. Calculated results are in agreement with previous experiments [18-20], i.e., transformation from starch to reducing sugar is blocked in lily bulbs during late storage. An intermediate product of starch degradation, soluble sugar is also a substrate involved in respiratory oxidation. The inflection point, the point at which $C_{\text {[sugar] }}$ is at a maximum, has always been used as a sign for dormancy release in the lily [21].

Water status has been thought to play a significant role in dormancy release in geophytes and has therefore been used as an indicator of dormancy [12,15]. It might thus be expected that MRI, with its acquired signal intensity sensitive to water status, might be a useful in vivo method of monitoring the dormancy release process. Compared with other imaging techniques, MRI is less invasive; it has high resolution and is sensitive to chemical composition changes within the organism. We measured $T_{1}, T_{2}$, and MTR param- 

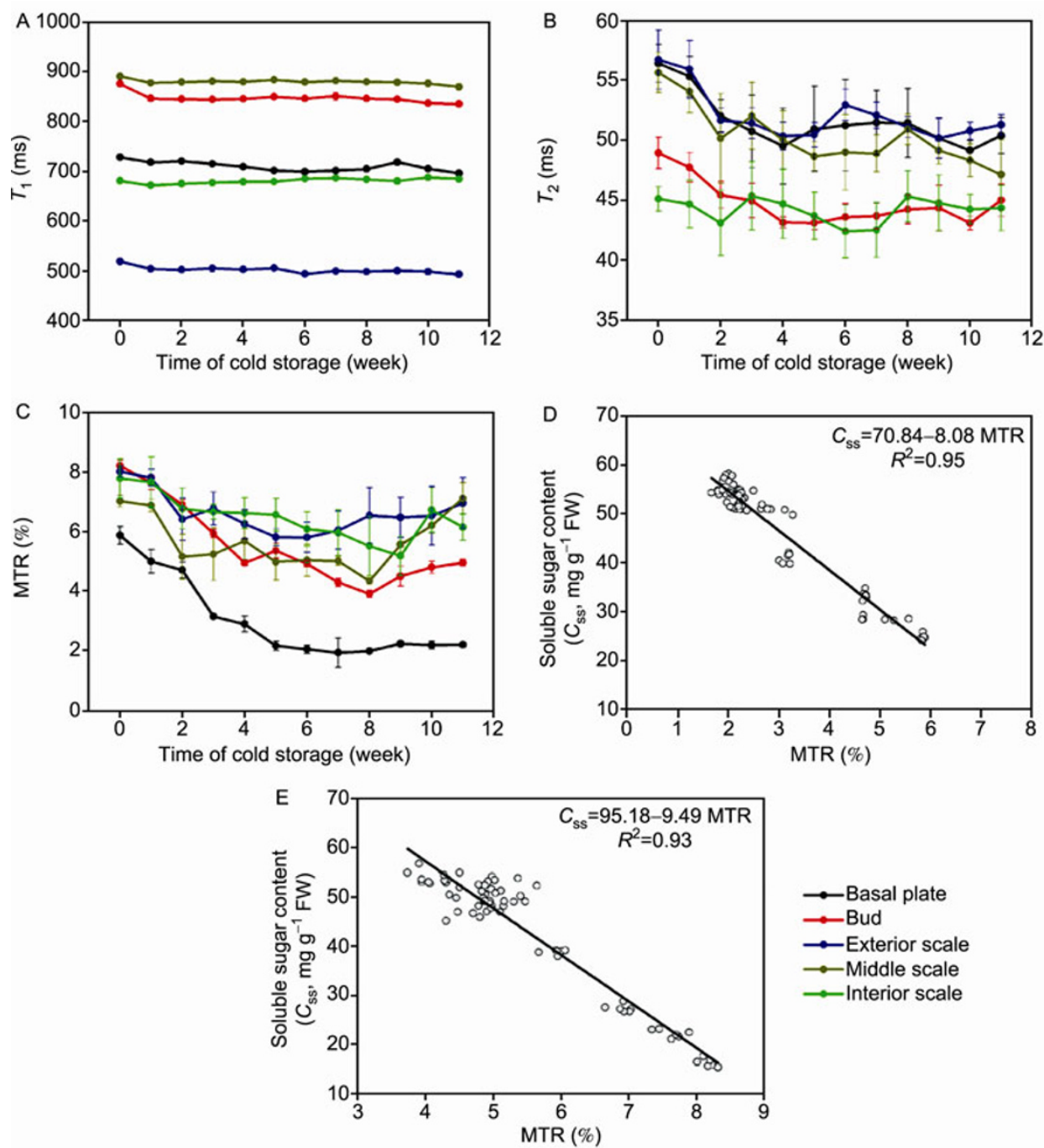

Figure 2 Parameters detected in the different lily bulb regions during 11 weeks of storage at $4^{\circ} \mathrm{C}$. Mean $( \pm \mathrm{SD}) T_{1}$ values obtained from different bulb regions are shown in A and the corresponding $T_{2}$ and MTR values are shown in B and C, respectively. The linear relationship between MTR and soluble sugar content was quantified in both the bud (D) and the basal plate (E) for the 11 week storage period $\left(R^{2}=0.95\right.$ for the bud, and $R^{2}=0.93$ for the basal plate)

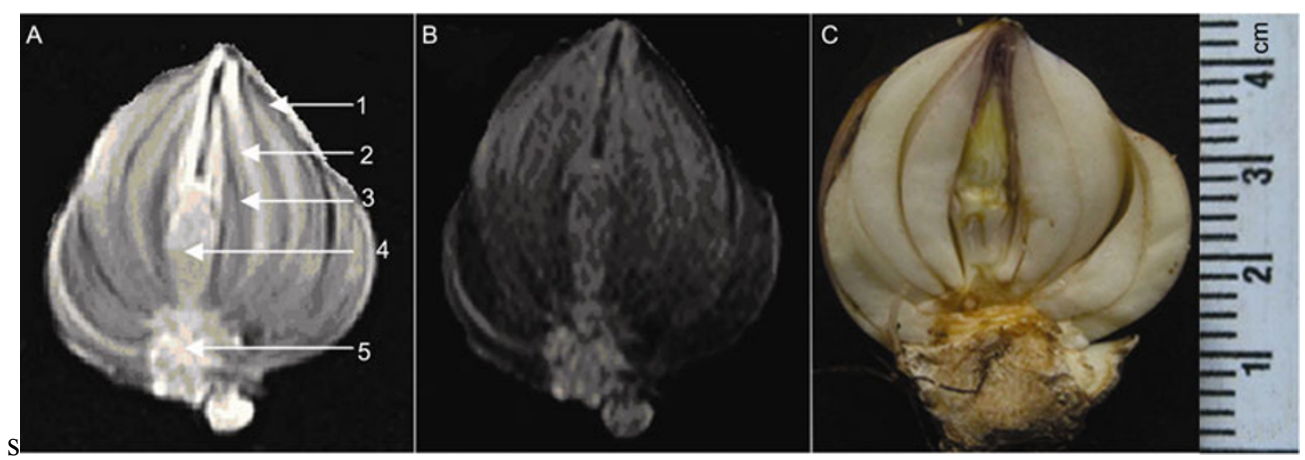

Figure 3 Characteristics of the performance of various sequences during the cold storage period. Region-of-interest in the slices (shown for the central slice) for the bulb of Lilium 'Sorbonne' is shown on $T_{1}(\mathrm{~A})$ and $T_{2}$-weighted (B) images after visualization during week 11 . The inner structure of the bulb is visible, which is composed of various scales: (1) exterior scale, (2) middle scale, (3) interior scale, (4) bud, and (5) basal plate. C, Lily bulb in vertical section. MR image quality was assessed by SNR: $T_{1}$-weighted $(0.97 \pm 0.01), T_{2}$-weighted $(0.56 \pm 0.20)($ paired $t$-test, $t=21.88, P<0.01)$.

eters over the course of bulb cold storage.

Relaxation times depend on several factors, such as proton mobility, observed spin, and presence of large mole- cules. In lily bulbs, the presence of abundant nonstructural carbohydrates including starch and soluble sugar increases sap viscosity. This process reduces water mobility and helps 
shorten MR relaxation times. Although continuous transformation of starch to soluble sugar takes place during bulb cold storage, $T_{1}$ and $T_{2}$ values did not appear to be shortened, and thus, cannot be considered as sensitive indicators for dormancy release in bulbs. The enhanced sap viscosity may contribute to the stable $T_{1}$ and $T_{2}$ values.

A decrease in total water content accompanies starch hydrolysis [22], resulting in an increase in both soluble sugar concentration and sap viscosity. This process reduces water mobility and is reflected in the shorter MR relaxation times found during later periods of cold storage. According to the equation $\mathrm{MTR}=(M-M s) / M$, an increase in bound water (relative to $M s$ ) and a decrease in total water content (relative to $M$ ) should result in a decrease in MTR values. Increased viscosity should also lead to a further decrease in MTR values, and thus MTR in the bud and basal plate could be considered as an alternative sensitive indicator for dormancy release in lily bulbs.

Not only is MRI sensitive to dormancy release in lily bulbs, but it also can be used to noninvasively monitor internal bulb structure. In general, bud elongation is the most important morphological change that occurs during dormancy break in lily bulbs. We obtained two image series of the bulb's internal morphology, and observed that better image quality of internal structures was obtained using the $T_{1}$ WIs. With a long $T E$, the magnetization vector was attenuated to such a weak level that the SNR of the $T_{2}$ WIs was much lower than that of $T_{1}$ WIs. In contrast, $T_{1}$ WIs and proton-weighted images with a shorter $T E$ were more feasible for monitoring the morphological changes.

Previous studies have shown that the physiological process of dormancy release in lilies involves complicated mechanisms associated with both morphological and metabolic changes. The process is regulated by many different factors [23]. In our study, changes in different MR relaxation parameters of stored bulbs were studied to evaluate the feasibility of monitoring metabolic changes during bulb dormancy using MRI. Based on the data presented in the discussion and our analysis, we conclude that (i) $T_{1}$ WIs have a greater SNR than $T_{2}$ WIs, and $T_{1}$ WIs are more suitable for in vivo morphological studies of the dormancy release process; (ii) MTR is sensitive to changes in $C_{\text {[sugar] }}$ during dormancy release; and (iii) MRI can detect the metabolic changes in lily bulbs in vivo during dormancy release, providing a new technological platform for studying the process and possessing great potential for evaluating the internal quality of flower bulbs. The information obtained in this study can serve as a guide to improve the quality of horticultural products.

This work was supported by the Special Fund for Agro-scientific Research in the Public Interest (Grant No. 200903020). The authors thank Dr. T. Fitzgibbon for comments on an earlier draft of the manuscript.
1 Langens-Gerrits M M, Nashimoto S, Croes A F, et al. Development of dormancy in different lily genotypes regenerated in vitro. Plant Growth Regul, 2001, 34: 215-222

2 Langens-Gerrits M M, Miller W B M, Croes A F, et al. Effect of low temperature on dormancy breaking and growth after planting in lily bulblets regenerated in vitro. Plant Growth Regul, 2003, 40: 267-275

3 Shin K S, Chakrabarty S D, Peak K Y. Sprouting rate, change of carbohydrate contents and related enzymes during cold treatment of lily bulblets regenerated in vitro. Scientia Hort, 2002, 96: 195-204

4 Yamazaki H, Nishijima T, Koshioka M, et al. Gibberellins do not act against abscisic acid in the regulation of bulb dormancy of Allium wakegi Araki. Plant Growth Regul, 2002, 36: 223-229

5 Xu R Y, Niimi Y, Han D S. Changes in endogenous abscisic acid and soluble sugars levels during dormancy-release in bulbs of Lilium rubellum. Scientia Hort, 2006, 111: 68-72

6 Gude H, Verbruggen J J M, Franssen J M, et al. Physiological markers for lily bulb maturity. Acta Hort, 2000, 517: 343-350

7 Sun H M, Li T L, Li Y F. Changes in free amino acid content and composition in lily bulb stored under cold temperature. Plant Physiol Commun, 2004, 40: 414-418

8 Han H B, Li K, Yan J H, et al. An in vivo study with an MRI tracer method reveals the biophysical properties of interstitial fluid in the rat brain. Sci China Life Sci, 2012, 55: 782-787

9 Roh M S. Flowering and inflorescence development of Lachenalia aloides 'Pearsonii' as influenced by bulb storage and forcing temperature. Scientia Hort, 2005, 104: 305-323

10 Fennell A, Line M J. Identifying differential tissue response in grape (Vitis riparia) during induction of endodormancy using nuclear magnetic resonance imaging. J Amer Soc Hort Sci, 2001, 126: 681-688

11 Han H J, Han H B, Sun X M, et al. Application of magnetic resonance imaging (MRI) technique on monitoring flower bud differentiation of tulip. J Nuclear Ag Sci, 2009, 23: 248-252

12 Zemah H, Bendel P, Rabinowitch H D, et al. Visualization of morphological structure and water status during storage of Allium aflatunense bulbs by NMR imaging. Plant Sci, 1999,147: 65-73

13 Van Kilsdonk M G, Nicolay K, Franssen J M, et al. Bud abortion in tulip bulbs studied by NMR imaging. J Exp Bot, 2002, 53: 1603-1611

14 Sardans J, Peñuelas J, Piedrafita S L. Changes in water content and distribution in Quercus ilex leaves during progressive drought assessed by in vivo ${ }^{1} \mathrm{H}$ magnetic resonance imaging. BMC Plant Biol, 2010, 10: 188

15 Okubo H, Iwaya-Inoue M, Motooka K, et al. Monitoring the cold requirements in tulip bulbs by 1H-NMR imaging. Acta Hort, 1997, 430: $411-417$

16 Van-As H. Intact plant MRI for the study of cell water relations membrane permeability, cell-to-cell and long-distance water transport. J Exp Bot, 2007, 58: 743-756

17 Bendel P, Zemah H, Kamenetsky R, et al. Magnetization transfer and double-quantum filtered imaging as probes for motional restricted water in tulip bulbs. Magn Reson Imaging, 2001, 19: 857-865

18 Miller W B, Langhans R W. Low temperature alters carbohydrate metabolism in Easter lily bulbs. HortScience, 1990, 25: 463-465

19 Tu S P, Mu D, Liu C. The Physiological and biochemical changes of lily bulbs during dormancy release with cold treatment. Acta Agricult Univ Jiangxiensis, 2005, 27: 404-407

20 Chen F, Hu X S. The effect of storage temperature on carbohydrate content and chip color of the potato tuber. Acta Hort Sin, 2000, 27: 218-219

21 Xu W W, Xia Y P. Morphological and physiological changes of oriental lily bulbs during dormancy release with low temperature storage. Northern Horticult, 2007, 9: 126-128

22 Sun X M, Li S, Luo F X. Regular patterns of water contents of bulbs of oriental hybrids lily during cold storage. J Liaoning Forest Sci Technol, 2008, 1: 13-15

23 Zhao L J, Su Y M. Present situation and develop management of floriculture industry in China. China Flowers Horticult, 2003, 2: 4-5

Open Access This article is distributed under the terms of the Creative Commons Attribution License which permits any use, distribution, and reproduction in any medium, provided the original author(s) and source are credited. 